\title{
Combined bacterial and viral treatment: a novel anticancer strategy
}

\author{
MARCIN P. KRZYKAWSKI
}

Department of Immunology, Jagiellonian University Medical College, Krakow, Poland

\begin{abstract}
An idea for a new combination therapy will be described herein. It is a proposition to combine viral and bacterial anticancer therapies and make them fight cancer in concert. We analyzed biological anticancer therapies and found overlapping advantages and disadvantages which led us to the conclusion that the combination therapy has the potential to create a new therapeutic quality. It is surprising how many weaknesses of viral anticancer therapy are the strengths of bacterial anticancer therapies and the other way round. We review the facts behind this concept and try to assess its value. We propose a few strategies how to combine these two therapies but as far as the review can go, final answers will have to come from the experiments. This review is the first attempt to describe a new strategy and understand the means for this idea but also to raise new questions and discuss new ways to look at anti-cancer treatment.
\end{abstract}

Key words: oncolytic bacteria, oncolytic viruses, anticancer therapy.

(Centr Eur J Immunol 2015; 40 (3): 366-372)

\section{Introduction}

Despite all the research and the countless attempts to find a perfect anti-cancer therapy, the most successful and effective therapy remains to be tumor resection. In the $19^{\text {th }}$ century clinicians observed that viral or bacterial infection [1] could lead to partial tumor regression [2] and sometimes, could even cure a patient. Limited understanding of microbiology, the discovery of radioactivity and later, first chemotherapies made the biological therapies less attractive. Bacteria and viruses remained in the shadow of the main stream of science until the critical advancement in microbiology and genetic engineering from the 1950s to the 1980s. In the meantime many chemotherapeutics were developed, isolated success stories gave some hope but were rarely confirmed in large clinical trials. At the dawn of chemotherapy, science moved to antibodies, developed gene therapy, immune therapy and gave a second chance to genetic engineering of microorganisms. Hundreds of proteins had been tested and a few were a minor breakthrough. Herceptin, an antibody against HER2 receptor, with relatively low side effects led to a substantial improvement in the patient's outcome. Unfortunately, it is only useful in some HER 2 positive breast cancer patients and a few other HER 2 positive cancers. Most immunotherapies which include bacteria or viruses have also failed except for one. Namely, Bacillus Calmette-Guerin (BCG) therapy is an accepted bacterial immunotherapy against non-invasive bladder cancer and it significantly decreases tumor recur- rence and metastases. Biological anti-cancer therapies still conceal its potential. Both bacterial and viral anti-cancer therapies have the hypothetical advantages which have never been found in any described therapy. In this review we want to propose a new strategy based on combinational bacterial and viral anti-cancer therapies. Both of them present advantages and disadvantages, but they fail to fight cancer when used separately. We will discuss how to couple these two therapies and how they support one another.

\section{Bacteria and viruses}

Since the very beginning of modern medicine in the $19^{\text {th }}$ century physicians observed tumor regression coinciding with infections. It was a time when the basics of microbiology were being settled and a distinction between bacteria and viruses was not so clear. Biological cancer therapies using viruses or bacteria proved to be beneficial to some patients but lost the struggle for attention with the rise of chemotherapy and radiotherapy. Half a century had to pass until microbiology was developed enough and genetic modification started to be possible. After many years of research on chemotherapy and radiotherapy scientists did not find the perfect drug. In the search of better therapies, science returned to the abandoned ideas. Using modern techniques improves previous weaknesses to carve potential therapies. Luckily both bacteria and viruses are

Correspondence: Marcin P. Krzykawski, MSc, Department of Immunology, Jagiellonian University Medical College, Krakow, Poland, e-mail: krzykawski.marcin@gmail.com 
fairly easy for genetic manipulation and there are plenty possible species to research.

\section{Viruses}

Reports of some patients with regressing tumors after some infections followed a similar, at that time unexplainable, pattern. Patients with advanced stages of cancer got infected and immediately after the infection, the tumor started to shrink. Unfortunately, remission did not last for more than a month. Finally, cancer progressed and patients died [3]. Today we have already learned that a rapid tumor regression followed by tumor progression means viral infection. Tumor relapse occurs because of the immune system producing antibodies that clear the patient of viruses. Today, even without any serious breakthrough, viruses tempt scientists with unique properties. Viruses can selectively kill tumor cells. It is possible thanks to two major strategies: a) replacing viral promoters with tumor specific promoters [4], and b) deleting the genes dispensable in cancer cell $[5,6]$. Prostate specific promoter has been used to create ARCA (CN706) virus which can divide only in cells with PSA (prostate specific antigen) expression. There are two important examples of oncoviruses with deleted genes: ONYX-015 with E1b-55k deletion (p53 binding site) and $\mathrm{Ad} \Delta 24$ without $\mathrm{Rb}$ binding site of E1A (described later). Onyx-015 mutant can kill p53 mutants, potentially more than $50 \%$ of tumors [7]. Cells with p53 mutations are resistant to hypoxia induced apoptosis $[8,9]$. Therefore, p53 mutants are even more important. It all started with ONYX-015. It has been a clinical failure but has opened a new door to the remarkable idea of CRAds, conditionally replicating adenoviruses. ONYX-015 had a deletion of E1b-55kDa. In a wild type E1b binds to an active p53 and inactivates it to allow viral replication. Without E1b the virus can replicate only in p53-cells. It makes the virus a perfect bullet for more than $50 \%$ of cancers with a p53 mutation. In recent years we could observe a few clinical trials. The most important one was ONYX-015. It reached the third stage of clinical trials but finally failed to prove its efficacy. Recently, in March 2013, T-VEC (herpes simplex virus 1 based therapy) has successfully passed phase three clinical trials.

\section{Bacteria}

William Coley was the first one to go beyond a sheer observation; he actually created a therapy based on attenuated bacteria. As other clinicians before him he also observed that in cancer patients, staying in beds that were previously used by patients with infectious diseases, the tumor regression has been observed. At that time people knew that diseases could be transmitted so the tumor regression had something to do with the previous patients. Coley isolated patient's bacteria and created a mix of two attenuated bacteria called Coley's Toxin. At that time it has been a successful therapy capable of reducing many tumors and, sometimes, leading even to a complete tumor eradication [10]. Unfortunately, nobody really knew why these therapies worked. Again, for the same reasons as viruses, bacterial anticancer therapy has been put aside. Presently, due to the development in microbiology and immune system field bacteria regained scientific attention. After many years of research on a variety of bacteria, Bacillus Calmette-Guerin (BCG), only one has been approved. The first and so far the only FDA-approved bacterial therapy (BCG) is an unspecific immune stimulator against bladder cancer [11]. Patients with non-muscle invasive bladder cancer, about $70 \%$ of bladder cancer patients, are treated with BCG. Bacillus Calmette-Guerin intravesical injection is preceded by endoscopic transurethral tumor resection and needs to be performed many times. The exact mechanism is not fully understood but it has been shown that NK cells [12] and cytotoxic T lymphocytes play a crucial role [13]. As a result, up to $60 \%$ of patients will become tumor free [14]. First of all, bacteria will be attracted to the hypoxic regions of tumors. Growing tumor cannot develop healthy vasculature and without one, hypoxic regions appear. Therefore, bacteria of choice are optional or obligatory anaerobes [15] which will limit the spread of the bacteria mainly to the tumor tissue. As a result of hypoxia and handicapped vascularization, many cells die leaving all the debris in the tumor. While choosing potential candidates for bacterial anti-cancer therapy it is also important to choose intracellular bacteria. In this review we will concentrate on Salmonella typhimurium [16] but Listeria monocytogenes [17] is also a very important candidate.

\section{Combined therapies}

\section{How can a combination of therapies improve the outcome?}

Both viruses and intracellular bacteria are evolutionary designed to infect the eukaryotic cells, including humans. Biological anticancer therapy aims to take advantage of these skills in order to kill cancer cells. Unlike any other, these skills tempt scientists to try to develop anticancer therapy. First of all, both organisms are self-replicating in the tumor milieu. As viruses need cells to replicate, they can find them in excess in the tumor. If we manage to deliver the therapeutic agent into the tumor, viruses and bacteria will replicate. It means that we can use lower doses of microorganisms and spare the other regions of the organism the toxicity and side effects common to other therapies. In clinical trials both therapies have been delivered by i.v. injections with a different efficiency. Unfortunately, viruses are very effectively cleared from blood by the innate mechanisms. For example, AdV-5 (adenovirus type 5), after i.v. injection binds to circulating platelets which aggregate and end up in liver sinusoids where they are de- 
graded by Kupfer cells [18-21]. As a result a viral half-life is about 2 minutes in mice [22] and goes up to 12 minutes in humans [23]. After secondary injection resulting in a production of antibodies these times get even shorter. On the other hand, bacteria, for example Salmonella typhimurium, have much longer half-life time ranging within 24 hours both in mice and humans [24, 25]. Animal research suggests also that in contrast to viruses bacteria can be re injected. After the first exposure to the pathogen, adaptive immunity also takes part in fighting pathogens using specific CTLs or antibodies. They are very effective means of clearing the blood of any pathogen. However, the delivery of therapeutic agent remains a problem because high doses may be detrimental and low doses will not provide sufficient concentration at the tumor. Due to the ability of active migration, bacteria are very well suited as a tumor delivery system [26]. Salmonella typhimurium has been modified to exhibit chemotaxis towards tumor. It involves modifications of the aspartate receptor and ribose receptor which direct bacteria into tumor tissue. There is also a serine receptor that makes bacteria prone to penetrate the host tissue $[16,27]$. Bacterial migration skills can be further exploited. Even in the case of initial good delivery, antiviral antibodies will reach the tumor cells and will clear them of viral particles. If we are able to attach the virus to the bacteria, intracellular bacteria will give a shelter to the viruses as well. Moreover, bacteria can also overcome another obstacle faced by viruses, tumor compartmentalization. Researchers working with oncolytic viruses observed that when virus reaches the tumor, it cannot spread into the entire tumor, if there are any fibrous barriers between tumor compartments. Surrounding the tumor with fibroblasts is one of the protective methods helping to slow down the tumor growth. Growing tumors often find a way out and bud. Such a bud creates a new compartment which is again surrounded by a fibrous tissue. Even if effectively delivered, viruses cannot cross these barriers and ultimately cannot kill the entire tumor. On the other hand, bacteria have the ability to actively migrate and have never been reported to have a problem in seeding the entire tumor. For example, Listeria monocytogenes can migrate between infected cells without being exposed to the immunological system [28]. Bacterial motility and natural ability to dissect fibrous tissue can help to seed the tumor and maximize virus distribution. Yet another problem shared by all intracellular therapies needs to be solved. In comparison to the surrounding healthy tissue, the tumor has an "increased hydrostatic pressure" [18]. Bacteria chemoattracted by cell debris, composed of necrotic parts of the tumor, can migrate against the current produced and by hiding inside the tumor cell will remain in the tumor while freely floating drugs as well as viruses are pushed out of the tumor. Motile bacteria can easily overcome the current and seed every tumor niche. The bacterial migratory skill comes with another therapeutic advantage, targeting me- tastases. It is still a matter of debate with counterarguments from different experiments but it has been shown that bacteria can migrate not only into the main tumor but also small metastatic niche [26, 29]. Eventually clinical trials uncover the clinical facts which did not really stand up to the promises seen at the beginning [26, 29]. Both of these features come from the bacterial tumor tropism. If bacteria are so much better why bother with viruses? Bacteria can be better at targeting the tumor but viruses have unprecedented killing skills. Viruses are not only very effective killing mechanisms but the most exciting fact is the ability of certain mutants to selectively kill cancer cells. Each type of viruses has its unique ability to attack eukaryotic cells. Mutants of HSV-1 and Parova virus need dividing cells $[30,31]$ while reovirus is dependent on an activated ras pathway [32]. After infection viral genetic material has to be reproduced inside the cell. The mechanism leading to the reproduction is strictly controlled by a host cell gene expression. In other words, viral genetic material will be expressed only if it contains promoters which can be activated by host cell machinery. Thanks to genetic engineering we can modify viruses so that they are able to attack only tumor cells. We believe that CRAds are the future of oncolytic viruses. Retinoblastoma protein $(\mathrm{Rb})$ is even a more promising weapon against cancer than Onyx-15 was. Retinoblastoma protein guards cell-cycle in normal cells. For the cancer cells to multiply, Rb needs to be constantly inactive (phosphorylated $\mathrm{Rb}=\mathrm{pRb}$ ). It can be achieved by a direct $\mathrm{Rb}$ mutation or mutation in one of many $\mathrm{Rb}$ activators among which we can find the deadliest oncogenes like: p53, p15, CDKs, cyclins, myc, ras and other crucial oncogenes. As a matter of fact, all human cancers have inactivated Rb. Mutated viruses with deleted E1a (Rb-binding and inactivating site) are unable to proliferate in healthy cells. Healthy cells are additionally protected by interferons. Since all types of cancer have to have inactivated $\mathrm{Rb}$ it means that not only $\mathrm{Ad} \Delta 24$ is selective to tumor cells but it also has the potential to kill all types of cancer. It makes $\operatorname{Ad} \Delta 24$ an ideal candidate for tumor eradication therapy. It is also worth mentioning that these oncogenes are called undruggable genes [33] and despite a lot of effort there are neither low molecular drugs nor proteins targeting these proteins. Moreover, healthy cells have an additional mechanism of protection against viral infection. Infected cell produces interferon (IFN)- $\alpha$ and IFN- $\beta$ [34] which acts as an alarm to the neighboring cells. To protect from viral infection neighboring cells shut down all the metabolic machinery necessary for the virus to replicate. Many cancer cells lack this mechanism and therefore are more susceptible to viruses. Viruses and bacteria can work in a feedback loop. While multiplying viruses lyse the tumor and release cell debris which in turn serve as a source of the nutrients for bacterial growth. It is also well known that problems in treating cancer come from cancer stem cells (CSC) which are often drug resistant. 
Bacteria and viruses are not specific to any particular antigen and cannot be pumped out of the cell as many drugs are. Therefore, biological therapies are equally useful against all cancer cells. Some CSCs are rapidly dividing, what makes them even more susceptible to viral infections. Another problem are senescent cancer cells which are responsible for tumor regression. These cells remain inactive and are very insensitive to any therapy, therefore contribute to cancer regression after becoming active again. $\mathrm{Vi}$ ruses will probably spare senescent cells but bacteria, by inducing strong immune response, help to clear the remaining cells. Additionally, intracellular bacteria may kill such a cell on its own.

\section{Immune reaction}

Tumors can be divided into immunogenic and non-immunogenic ones [35]. Immunogenic tumors are partly susceptible to immunotherapy but, unfortunately, tumor immunosuppression and evolution attenuate immune response resulting in tumor recurrence. On the other hand, we can treat non-immunogenic tumors with chemotherapy or radiotherapy which, carefully designed, can lead to cancer remission. Again, cancer still holds the advantage by metastasizing and evolving out of therapy killing spectrum $[36,37]$. All tumors are somewhere on the range between those two. The role of bacteria as a immunostimulant is to amplify the strength of the immune reaction leading to better immunotherapy [38]. Pre-existing immunity can be amplified by a cocktail of cytokines like interleukin (IL)-2 IFN- $\alpha$, IFN- $\beta$ or IFN- $\gamma$, IL-12, IL-23, GM-CSF and others. It is clear when we talk about more immunogenic tumors but what happens with less immunogenic tumors? Since we are proposing using two pathogens we also expect to amplify the reaction against viral antigens expressed on cancer cells infected by viruses and expressing E1A antigen [39]. Cells infected by viruses expose E1A in MHC what makes the cell immunogenic and marks it to be killed by the immune system [40]. It is a well-established mechanism that cells infected by viruses release interferons IFN to warn neighboring cells about threats. It has also been shown that Salmonella typhimurium infection can give similar results and release of IFN from macrophages [41] leading to necroptosis [42-44] and pyroptosis of macrophages [45]. Moreover, some type of interferons can additionally promote an antitumor immune response by recruiting and stimulating NK cells [46].

For final tumor eradication it is not bacteria that has the final word against the tumor. Syngenic tumors in mice injected with bacteria regressed. Shortly after tumor disappeared, mice were already bacteria free and the tumor shrinkage continued until it was completely cleared out [47]. Not were mice only tumor free, they were immunized against tumor antigens. It means that bacterial anticancer therapy is based on bacteria killing cancer cells in this or the other way but also bacteria inducing strong host immune response against cancer cells.

\section{Threats}

Before deciding to infect a patient with a pathogen we need to consider the risks of using an experimental therapy based on pathogens versus the probability of successful treatment using standard therapy. This question becomes even more important when one considers the use of two pathogens. Until we do not know the real reaction of organisms, this kind of combination should be used only when current therapies give no real hopes. Clinical data on oncolytic bacteria give no reasons to suspect the threats of sepsis but it can never be ruled out so to protect the patient we will have to use only bacteria susceptible to antibiotics. Similarly with viruses; the immune system can efficiently clear the virus out but for the safety it is important to be ready to use antiviral serum. Up till now no clinical data have shown serious side effects of bacterial or viral therapies. Nevertheless, we have to keep in mind experiments done on mice. By accumulating in hypoxic regions of tumors, bacteria gain perfect growing conditions. Murine experiments showed that injection of Clostridium botulinum spores into tumor bearing mice may be lethal [15, 48]. Luckily, there are many potentially therapeutic bacteria to choose from. For example, Salmonella typhimurium can equally well accumulate in tumors but while growing keep the toxicity low [49]. Another line of defense is the genetic manipulation which prohibits viruses and bacteria to infect any other tissue besides the tumor. To insure the safety of these mutations and prevent reversed mutations it is important to use proper genetic techniques, like deleting genes instead of mutating a part of the gene. We can do a lot to prevent all the predicted complications but we also stay with an open question how our organisms will react to such a coinfection and it will be answered only by experiment. Because of the coinfection risk [50], the final therapy will have to be carefully tailored.

Up-to-date experience with neither of these therapies showed severe side effects [51], or even comparable to those observed for chemo- or radiotherapy.

\section{How to merge viral and bacterial therapies?}

By extracting the best out of two therapies we want to maximize the therapeutic result. We propose using bacteria as a carrier for viruses. Each pathogen has its role to play. Bacteria's main task is to deliver the virus into the tumor and by active migration hide it from the immune system. To make it possible the virus will have to be bound or fused into the bacteria for example with binding proteins like anti-viral scFv regions expressed on bacterial surface. 
If the virus is attached to bacteria they will hide together so that the immune system will not clear them out. By co-infecting the tumor we would allow viruses to spread inside the tumor and use their pinpoint ability to kill cancer cells. Since viruses kill by starting a lytic cycle which ends up with cell lysis and releasing of about 200 [52] new viruses which subsequently infect neighboring cancer cells every lytic cycle, which takes 40 to 48 hours [52]. Sooner or later the immune system will develop antibodies against viruses. But till then we hope to have the virus well spread and hidden together with bacteria. Very recently this idea has been partly supported by research done by Cronin et al. [53]. They have combined a non-pathogenic bacteria Escherichia coli expressing B18R, with a vesicular stomatitis virus (VSV). Bacteria were used to precondition the tumor by expressing B18R an antagonist of type I interferon. Blocking type I interferon communication resulted in a reduction in tumor growth and prolonged mice survival [53]. More supporting data come from clinical and microbiological experience. It has been known for decades that bacterial and viral coinfection result in a much higher morbidity and mortality [54-57].

\section{Conclusions}

The idea of combinational therapy presented here describes unknown possibilities. Both anti-tumor agents can act as long as the tumor exists. They survive thanks to the most conservative hallmark of cancer, the necrotic, immunosuppressed and hypoxic region of tumor. Unlike any drug, viruses can effectively kill cancer cells possessing no immunogenic mutations of nuclear proteins: p53 and $\mathrm{RB}[6,58]$. Both $\mathrm{p} 53$ and $\mathrm{Rb}$ are among so-called undruggable genes, so that neither low molecular drug nor a protein was found which could kill cells with those mutations. Modified viruses have to replicate in such cells to survive and cannot do so in non-mutated cells. Viruses can kill cancer cells without the contribution of the immune system. While viruses selectively attack and kill tumor cells, bacteria not only disseminate the virus inside the tumor, but they also induce a strong immune response against tumor antigens [59]. By inducing a strong immune response against bacterial antigens, they may overcome the immunosuppression produced by tumor cells and tumor supporting immune cells: TIL's, MDSC, TAM and TAN's, resulting in prevailing Th 1 type response [11]. This effect can be even stronger when supported by an antiviral response which would occur due to the presence of viruses. Properly designed bacteria can support this struggle by direct killing as much as by production of therapeutic proteins, e.g. proapoptotic proteins. These actions may possibly be even stronger due to the fact that we are dealing with self-replicating agents. Most of our arguments supporting the idea of combinational therapy is a result of scientific literature analysis. Very recently there has been an article based on a very similar idea where authors proved that such a therapy can lead to better results [53].

Safety issues remain a very important part of the discussion. Both therapies proved to be safe with no or mild side effects but very little is known about possible results in a combination therapy. Many crucial questions have to be answered: what is the risk that the infection will spread? Is there any risk to develop sepsis? Is there a risk of mutations which will turn the therapeutic agents against the patient? Do we have working retreat strategies in case infections would break out of control? These and other questions need to be answered before any serious attempts for clinical trials are introduced. Nevertheless, it will have to be thoroughly investigated how the human organism reacts to double infection $[51,60]$.

Author would like to thank professor Janusz Marcinkiewicz for his help and invaluable support in writing about bacterial anticancer therapies and doctor Ramon Alemany for his critical reading of the manuscript and help in understanding oncolytic viral therapy. The project operated within the Foundation for Polish Science VENTURES Programme co-financed by the European Regional Development Fund, Operational Program Innovative Economy 2007-2013 and INTER Programme co-financed by the European Social Fund. Publication costs were covered by K/ZDS/004602.

The author declares no conflict of interest

\section{References}

1. Tsung K, Norton JA (2006): Lessons from Coley's Toxin. Surg Oncol 15: 25-28. Available from: http://www.ncbi.nlm. nih.gov/pubmed/16814541 (Accessed 11 January 2015).

2. Kelly E, Russell SJ (2007): History of oncolytic viruses: genesis to genetic engineering. Mol Ther 15: 651-659. Available from: http://www.ncbi.nlm.nih.gov/pubmed/17299401.

3. Dock G (1904): The influence of complicating diseases upon leukćmia. Am J Med Sci 127: 563-592.

4. Rodriguez R, Schuur ER, Lim HY, et al. (1997): Prostate attenuated replication competent adenovirus (ARCA) CN706: a selective cytotoxic for prostate-specific antigen-positive prostate cancer cells. Cancer Res 57: 2559-2563.

5. Barker DD, Berk AJ (1987): Adenovirus proteins from both E1B reading frames are required for transformation of rodent cells by viral infection and DNA transfection. Virology 156: 107-121.

6. Fueyo J, Gomez-Manzano C, Alemany R, et al. (2000): A mutant oncolytic adenovirus targeting the $\mathrm{Rb}$ pathway produces anti-glioma effect in vivo. Oncogene 19: 2-12.

7. Hollstein M, Sidransky D, Vogelstein B, Harris CC (1991): p53 mutations in human cancers. Science 253: 49-53.

8. Graeber TG, Osmanian C, Jacks T, et al. (1996): Hypoxia-mediated selection of cells with diminished apoptotic potential in solid tumours. Nature 379: 88-91.

9. Yu JL, Rak JW, Coomber BL, et al. (2002): Effect of p53 status on tumor response to antiangiogenic therapy. Science 295: 1526-1528. 
10. Starnes CO (1992): Coley's toxins in perspective. Nature 357: 11-12.

11. Kawai K, Miyazaki J, Joraku A (2013): Bacillus CalmetteGuerin (BCG) immunotherapy for bladder cancer: current understanding and perspectives on engineered $B C G$ vaccine. Cancer Sci 104: 22-27.

12. Brandau S, Riemensberger J, Jacobsen M, et al. (2001): NK cells are essential for effective BCG immunotherapy. Int J Cancer 92: 697-702.

13. Ratliff TL, Gillen D, Catalona WJ (1987): Requirement of a thymus dependent immune response for BCG-mediated antitumor activity. J Urol 137: 155-158.

14. Shelley MD, Kynaston H, Court J, et al. (2001): A systematic review of intravesical bacillus Calmette-Guérin plus transurethral resection vs transurethral resection alone in Ta and T1 bladder cancer. BJU Int 88: 209-216.

15. Dang LH, Bettegowda C, Huso DL, et al. (2001): Combination bacteriolytic therapy for the treatment of experimental tumors. Proc Natl Acad Sci U S A 98: 15155-15160.

16. Kasinskas RW, Forbes NS (2006): Salmonella typhimurium specifically chemotax and proliferate in heterogeneous tumor tissue in vitro. Biotechnol Bioeng 94: 710-721.

17. Wood LM, Paterson Y (2014): Attenuated Listeria monocytogenes: a powerful and versatile vector for the future of tumor immunotherapy. Front Cell Infect Microbiol 4: 51.

18. Pesonen S, Kangasniemi L, Hemminki A (2011): Oncolytic adenoviruses for the treatment of human cancer: focus on translational and clinical data. Mol Pharm 8: 12-28.

19. Shayakhmetov DM, Li ZY, Ni S, Lieber A (2004): Analysis of adenovirus sequestration in the liver, transduction of hepatic cells, and innate toxicity after injection of fiber-modified vectors. J Virol 78: 5368-5381.

20. Tao N, Gao GP, Parr M, et al. (2001): Sequestration of adenoviral vector by Kupffer cells leads to a nonlinear dose response of transduction in liver. Mol Ther 3: 28-35.

21. Vetrini F, Ng P (2011): Liver-directed gene therapy with helper-dependent adenoviral vectors: current state of the art and future challenges. Curr Pharm Des 17: 2488-2499.

22. Alemany R, Suzuki K, Curiel DT (2000): Blood clearance rates of adenovirus type 5 in mice. J Gen Virol: 2605-2609.

23. Reid T, Galanis E, Abbruzzese J, et al. (2002): Hepatic arterial infusion of a replication-selective oncolytic adenovirus (d11520) phase ii viral, immunologic, and clinical endpoints. Cancer Res 33: 6070-6079.

24. Clairmont C, Lee KC, Pike J, et al. (2000): Biodistribution and genetic stability of the novel antitumor agent VNP20009, a genetically modified strain of Salmonella typhimurium. J Infect Dis 181: 1996-2002.

25. Toso JF, Gill VJ, Hwu P, et al. (2002): Phase I study of the intravenous administration of attenuated Salmonella typhimurium to patients with metastatic melanoma. J Clin Oncol 20: $142-152$.

26. Ganai S, Arenas RB, Sauer JP, et al. (2011): In tumors Salmonella migrate away from vasculature toward the transition zone and induce apoptosis. Cancer Gene Ther 18: 457-466.

27. Kasinskas RW, Forbes NS (2007): Salmonella typhimurium lacking ribose chemoreceptors localize in tumor quiescence and induce apoptosis. Cancer Res 67: 3201-3209.

28. Ireton K, Rigano LA, Polle L, Schubert WD (2014): Molecular mechanism of protrusion formation during cell-to-cell spread of Listeria. Front Cell Infect Microbiol 4: 21

29. Patyar S, Joshi R, Byrav DS, et al. (2010): Bacteria in cancer therapy: a novel experimental strategy. J Biomed Sci 17: 21.
30. Haag A, Menten P, Van Damme J, et al. (2000): Highly efficient transduction and expression of cytokine genes in human tumor cells by means of autonomous parvovirus vectors; generation of antitumor responses in recipient mice. Human Gene Ther 11: 597-609.

31. Yoon SS, Nakamura H, Carroll NM, et al. (2000): An oncolytic herpes simplex virus type 1 selectively destroys diffuse liver metastases from colon carcinoma. FASEB J 14: 301311.

32. Coffey MC, Strong JE, Forsyth PA, Lee PW (1998): Reovirus therapy of tumors with activated Ras pathway. Science 282: 1332-1334.

33. Yan C, Higgins PJ (2013): Drugging the undruggable: Transcription therapy for cancer. Biochim Biophys Acta 1835: 76-85.

34. Stein SC, Falck-Pedersen E (2012): Sensing adenovirus infection: activation of interferon regulatory factor 3 in RAW 264.7 cells. J Virol 86: 4527-4537.

35. Blankenstein T, Coulie PG, Gilboa E, Jaffee EM (2012): The determinants of tumour immunogenicity. Nat Rev Cancer 12: 307-313.

36. Almendro V, Cheng YK, Randles A, et al. (2014): Inference of tumor evolution during chemotherapy by computational modeling and in situ analysis of genetic and phenotypic cellular diversity. Cell Rep 6: 514-527.

37. Nowell PC (1976): The clonal evolution of tumor cell populations. Science 194: 23-28.

38. Schafer R, Portnoy DA, Brassell SA, Paterson Y (1992): Induction of a cellular immune response to a foreign antigen by a recombinant Listeria monocytogenes vaccine. J Immunol 149: 53-59.

39. Sparer TE, Wynn SG, Clark DJ, et al. (1997): Generation of cytotoxic $\mathrm{T}$ lymphocytes against immunorecessive epitopes after multiple immunizations with adenovirus vectors is dependent on haplotype. J Virol 71: 2277-2284.

40. Alemany R (2012): Design of improved oncolytic adenoviruses. 1st ed. Elsevier Inc. (online). Available from: http://www. ncbi.nlm.nih.gov/pubmed/23021243 (Accessed 11 January 2015).

41. Sing A, Merlin T, Knopf HP, et al. (2000): Bacterial induction of beta interferon in mice is a function of the lipopolysaccharide component. Infect Immun 68: 1600-1607.

42. Auerbuch V, Brockstedt DG, Meyer-Morse N, et al. (2004): Mice lacking the type I interferon receptor are resistant to Listeria monocytogenes. J Exp Med 200: 527-533.

43. O'Connell RM, Saha SK, Vaidya SA, et al. (2004): Type I interferon production enhances susceptibility to Listeria monocytogenes infection. J Exp Med 200: 437-445.

44. Robinson N, McComb S, Mulligan R, et al. (2012): Type I interferon induces necroptosis in macrophages during infection with Salmonella enterica serovar Typhimurium. Nat Immunol 13: 954-962.

45. Fink SL, Cookson BT (2007): Pyroptosis and host cell death responses during Salmonella infection. Cell Microbiol 9: 2562-2570.

46. Goldszmid RS, Dzutsev A, Trinchieri G (2014): Host immune response to infection and cancer: unexpected commonalities. Cell Host Microbe 15: 295-305.

47. Agrawal N, Bettegowda C, Cheong I, et al. (2004): Bacteriolytic therapy can generate a potent immune response against experimental tumors. Proc Natl Acad Sci U S A 101: 1517215177. 
48. Malmgren RA, Flanigan CC (1955): Localization of the vegetative form of Clostridium tetani in mouse tumors following intravenous spore administration. Cancer Res 15: 473-478.

49. Forbes NS (2010): Engineering the perfect (bacterial) cancer therapy. Nat Rev Cancer 10: 785-794.

50. Minton K (2013): Infection: resistance is futile. Nat Rev Immunol 13: 391.

51. Maciag PC, Radulovic S, Rothman J (2009): The first clinical use of a live-attenuated Listeria monocytogenes vaccine: A Phase I safety study of Lm-LLO-E7 in patients with advanced carcinoma of the cervix. Vaccine 27: 3975-3983.

52. Wigand R, Kümel G (1977): The kinetics of adenovirus infection and spread in cell cultures infected with low multiplicity. Arch Virol 54: 177-187.

53. Cronin M, Le Boeuf F, Murphy C, et al. (2014): Bacterial-mediated knockdown of tumor resistance to an oncolytic virus enhances therapy. Mol Ther 22: 1188-1197.

54. Jamieson AM, Pasman L, Yu S, et al. (2013): Role of tissue protection in lethal respiratory viral-bacterial coinfection. Science 340: 1230-1234.

55. Minton K (2013): Infection: resistance is futile. Nat Rev Immunol 13: 391.

56. Nichol KP, Cherry JD (1967): Bacterial-viral interrelations in respiratory infections of children. N Engl J Med 277: 667672.

57. Smith AM, Adler FR, Ribeiro RM, et al. (2013): Kinetics of coinfection with influenza A virus and Streptococcus pneumoniae. PLoS Pathog 9: e1003238.

58. Alemany R (2007): Cancer selective adenoviruses. Mol Aspects Med 28: 42-58.

59. Paterson Y, Guirnalda PD, Wood LM (2010): Listeria and Salmonella bacterial vectors of tumor-associated antigens for cancer immunotherapy. Semin Immunol 22: 183-189.

60. Choi JW, Lee JS, Kim SW, Yun CO (2012): Evolution of oncolytic adenovirus for cancer treatment. Adv Drug Deliv Rev 64: 720-729. 